\title{
Sur la fonction d'appui des ensembles convexes dans un espace localement convexe
}

\author{
Par LaRs Hörmander
}

\begin{abstract}
Cette note a son origine dans RÅDsTröm [4], où l'on démontre que certaines classes d'ensembles convexes dans un espace normé $E$, munies de la définition usuelle de l'addition et de la multiplication avec des scalaires et distanciées par la distance de Hausdorff, sont isomorphes à des cônes convexes dans un espace normé $N$.

Si la dimension de $E$ est finie, ce théorème est essentiellement dû à Minkowski, car un tel isomorphisme est donné par la correspondence entre les ensembles convexes et leurs fonctions d'appui, qui sont définies dans l'espace dual $E^{\prime}$ de $E$ et normées par le maximum sur la sphère unité dans $E^{\prime}$.

Nous allons démontrer ici que les théorèmes classiques sur la fonction d'appui sont encore valables si la dimension de $E$ n'est pas nécessairement finie. Les résultats n'offrent peut-être pas beaucoup de nouveau, mais autant que nous le savons, les démonstrations ne se trouvent nulle part. En appliquant les résultats démontrés par la suite nous obtiendrons une généralisation et une précision du théorème cité de Rå.dström.
\end{abstract}

Soit $E$ un espace vectoriel topologique localement convexe réel. Nous désignerons par $E^{\prime}$ l'espace dual de $E$, et la forme linéaire $y \in E^{\prime}$ sera écrite $\langle x, y\rangle(x \in E)$. De la théorie des espaces localement convexes nous citons le lemme suivant:

Lemme. Dans un espace localement convexe, tout ensemble convexe fermé $K$ est l'intersection des demi-espaces fermés qui le contiennent.

Rappelons qu'un demi-espace fermé est défini par une inégalité de la forme $\langle x, y\rangle \leq \alpha$, dans laquelle $y \in E^{\prime}$ et $\alpha$ est réel. - Le lemme se trouve dans BourBAKI [1], p. 73, Corollaire 1 .

Définition. Etant donné un ensemble convexe fermé non vide $K$ dans $E$ on définit sa fonction d'appui $H(y), y \in E^{\prime}$, par

$$
H(y)=\sup _{x \in K}\langle x, y\rangle
$$

$H(y)$ est évidemment partout $>-\infty$. Rappelons qu'un ensemble $A \subset E$ est faiblement borné si $\langle x, y\rangle$ est borné quand $x \in A$ pour chaque $y$ fixé dans $E^{\prime}$. 


\section{HörmaNDER, Sur la fonction d'appui}

On voit alors immédiatement de (1) qu'un ensemble convexe et fermé $K$ est faiblement borné si et seulement si la fonction d'appui $H(y)$ est finie pour chaque $y \in E^{\prime}$.

Théorème 1. Si les fonctions d’appui de deux ensembles convexes fermés $K_{1}$ et $K_{2}$ sont identiques on a aussi $K_{1}=K_{2}$.

L'hypothèse peut s'écrire comme il suit: Si $\langle x, y\rangle \leq \alpha$ pour $x \in K_{1}$ on a aussi $\langle x, y\rangle \leq \alpha$ pour $x \in K_{2}$ et réciproquement. Autrement dit, un demi-espace fermé qui contient un des deux ensembles en contient aussi l'autre. Il s'ensuit alors du lemme que $K_{1}$ et $K_{2}$ sont identiques.

Théorème 2. Si $K_{1} \subset K_{2}$ on $a H_{1} \leq H_{2}$ et réciproquement.

La partie directe est triviale, et la réciproque s'ensuit du lemme en appliquant le même raisonnement que précédemment.

On définit habituellement la somme de deux ensembles convexes $K_{1}$ et $K_{2}$ par $K_{1}+K_{2}=\left\{x_{1}+x_{2} ; x_{i} \in K_{i}\right\}$. Ici cette définition n'est pas convenable puisque la somme ainsi définie n'est pas nécessairement fermée si $K_{1}$ et $K_{2}$ sont fermés, même si la dimension de $E$ est finie. Nous posons

$$
K_{1}+K_{2}=\overline{\left\{x_{1}+x_{2} ; x_{i} \in K_{i}\right\}}
$$

où le trait désigne l'adhérence. La multiplication avec un scalaire non négatif. $t$ est définie de la manière usuelle,

$$
t K=\{t x ; x \in K\}
$$

Théorème 3. Si les fonctions d'appui de $K_{1}$ et $K_{2}$ sont $H_{1}$ et $H_{2}$, la fonction d'appui de $t_{1} K_{1}+t_{2} K_{2}$ est $t_{1} H_{1}+t_{2} H_{2}\left(t_{1}, t_{2} \geq 0\right)$. Ici il faut adopter la convention $0 \cdot \infty=0$.

Puisque les points $t_{1} x_{1}+t_{2} x_{2}, x_{i} \in K_{i}$, sont denses dans $t_{1} K_{1}+t_{2} K_{2}=K$ on a en effet

$\sup _{x \in K}\langle x, y\rangle=\sup _{x_{i} \in \Pi_{i}}\left\langle t_{1} x_{1}+t_{2} x_{2}, y\right\rangle=t_{1} \sup _{x_{1} \in K_{1}}\left\langle x_{1}, y\right\rangle+t_{2} \sup _{x_{2} \in K_{2}}\left\langle x_{2}, y\right\rangle=t_{1} H_{1}(y)+t_{2} H_{2}(y)$.

L'enveloppe convexe fermée des ensembles convexes fermés $K_{\imath}(\iota \in I)$ est l'adhérence de l'ensemble des combinaisons linéaires $\sum_{I} \lambda_{\imath} x_{\imath}$, où $x_{\imath} \in K_{\iota}, \lambda_{\imath} \geq 0$ pour tout $\iota \in I\left(\lambda_{t}=0\right.$ sauf pour un nombre fini d'indices) et $\sum_{I} \lambda_{\iota}=1$. Il en découle par un raisonnement analogue à celui, utilisé dans la démonstration du théorème 3 , le théorème suivant:

Théorème 4. La fonction d'appui de l'enveloppe convexe fermée de $K_{\imath}(\iota \in I)$ est $\sup _{1} H_{\imath}(y)$.

Nous démontrerons maintenant un théorème plus difficile qui caractérise entièrement les fonctions d'appui.

Théorème 5. Une fonction $H(y)$ définie dans $E^{\prime},-\infty<H(y) \leq+\infty$, est la fonction d'appui d'un ensemble convexe fermé dans $E$ si et seulement si $H(y)$ est 
semicontinu inférieurement pour la topologie faible $\sigma\left(E^{\prime}, E\right)$ et de plus convexe et positivement homogène, ce qui s'écrit

$$
H\left(y_{1}+y_{2}\right) \leq H\left(y_{1}\right)+H\left(y_{2}\right), H(t y)=t H(y) \quad(t \geq 0) .
$$

Pour la notation $\sigma\left(E^{\prime}, E\right)$ et les théorèmes utilisés plus loin voir DieUDONNÉ [2], [3].

Les conditions sont nécessaires. En effet, pour $x$ fixé dans $E,\langle x, y\rangle$ est une fonction de $y$ qui est linéaire, homogène et continu pour la topologie faible. Donc $H(y)$, étant le suprémum d'une famille de fonctions de ce genre, est convexe, positivement homogène et semicontinu inférieurement pour la topologie faible.

Il est plus difficile à établir que la condition soit suffisante. Nous allons nous servir d'une méthode qui est bien connue dans le cas où la dimension de $E$ est finie (voir SANDGREN [5]). Notre mérite est donc seulement d'avoir trouvé les conditions topologiques appropriées. ${ }^{1}$

Posons $F=E+R$, où $R$ est un espace vectoriel à une dimension. Les éléments de $F$ sont de la forme $X=(x, \xi)$, où $x \in E$ et $\xi$ est un nombre réel, et la topologie de $F$ est la topologie produit. L'espace dual $F^{\prime}$ est évidemment réalisé par $E^{\prime}+R$, et nous pouvons écrire pour $X \in F$ et $Y \in F^{\prime}$

$$
\langle X, Y\rangle=\langle x, y\rangle-\xi \eta
$$

A un ensemble convexe fermé $K$ dans $E$ nous faisons correspondre dans $F$ le cône convexe $C$ défini par

$$
C=\overline{\{(t x, t) ; x \in K, t \geq 0\}}
$$

L'interprétation géométrique de cette définition est que $C$ est le cône dont la base est $\{(x, 1) ; x \in K\}$ et le sommet à l'origine. On voit immédiatement que tous les points $X \in C$ sont de la forme $(t x, t)$ sauf pour ceux qui sont situés dans le plan $\xi=0$. Donc on conclut qu'il y a une correspondence biunivoque entre les ensembles convexes fermés $K$ dans $E$ et les cônes convexes fermés $C$ dans $F$, qui ont leur sommet à l'origine et qui sont contenus dans le demiespace $\xi \geq 0$ mais non dans le plan $\xi=0$.

Considérons dans $F^{\prime}$ le cône dual $C^{\prime}$ qui est défini par

$$
C^{\prime}=\{Y ;\langle X, Y\rangle \leq 0 \text { pour } X \in C\} .
$$

Cette définition peut s'écrire $\langle x, y\rangle-\eta \leq 0$ pour $x \in K$, c'est-à-dire $\eta \geq H(y)$. Donc $C^{\prime}$ est l'image géométrique de la fonction d'appui de $K$.

Prenons maintenant une fonction $H(y)$ qui satisfait aux conditions de l'énoncé $\mathrm{du}$ théorème 5 et posons:

$$
D=\{Y ; \eta \geq H(y)\} .
$$

1 Voir aussi Fenchex: On conjugate convex functions, Canad. Journ. of Math. 1, 73-77, où l'on pose une condition de semicontinuité dans l'étude de la transformation de Tegendre généralisée à un nombre fini de dimensions. 


\section{Hörma NDER, Sur la fonction d'appui}

Les conditions (4) entraînient que $D$ est un cône convexe, et le fait, que $H$ est faiblement semicontinu inférieurement, entraîne que $D$ est fermé pour la topologie faible. Du fait que $H(0)=0$ il s'ensuit que $(0, \eta) \in D$ seulement si $\eta \geq 0$.

Soit $C$ le cône dual de $D$,

$$
C=\{X ;\langle X, Y\rangle \leq 0 \text { pour tout } Y \in D\}
$$

Etant l'intersection de demi-espaces fermés, $C$ est un cône convexe fermé. $C$ est situé dans le demi-espace $\xi \geq 0$, car $(0, \eta) \in D$ si $\eta \geq 0$. Il est clair que $C^{\prime} \supset D$. Si nous démontrons que $C^{\prime}=D$ il s'ensuivra d'abord que $C$ n'est pas contenu dans le plan $\xi=0$, car alors le point $(0,-1)$, par exemple, appartiendrait à $D$ ce qui est une contradiction. Donc l'ensemble convexe fermé $K$ dans $E$, qui correspond à $C$, a la fonction d'appui $H(y)$.

Il reste seulement à démontrer que $C^{\prime} \subset D$, ou ce qui est la même chose, qu'étant donné $Y_{0} \notin D$ il existe $X \in C$ tel que $\left\langle X, Y_{0}\right\rangle>0$. Autrement dit, il faut démontrer que si $Y_{0} \notin D$ il existe un élément $X \in F$ tel que $\left\langle X, Y_{0}\right\rangle>0$ et. $\langle X, Y\rangle \leq 0$. pour tout $Y \in D$. Ceci s'ensuit facilement du lemme. En effet, si on munit $F^{\prime}$ de la topologie faible, les fonctionnelles linéaires sur $F^{\prime}$ peuvent s'écrire $\langle X, Y\rangle$ avec $X \in F$ (voir Dieudonné [2]). Donc d'après le lemme il existe un élément $X \in F$ et un nombre réel $\alpha$ tels que $\left\langle X, Y_{0}\right\rangle>\alpha$ et $\langle X, Y\rangle \leq \alpha$ pour $Y \in D$. Etant donné que $0 \in D$, il s'ensuit que $\alpha \geq 0$, donc $\left\langle X, Y_{0}\right\rangle>0$. Puisque $D$ est un cône on a également $\langle X, t Y\rangle \leq \alpha$ pour tout $t>0$, et il en découle que $\langle X, Y\rangle \leq 0$.

Le théorème suivant est un complément de notre théorème 5 .

Théorème 6. La fonction d'appui de $K$ est continue pour la topologie faible si et seulement si $K$ est borné et de dimension finie.

Les ensembles $U=\left\{y ;\left|\left\langle x_{i}, y\right\rangle\right|\langle 1, i=1, \ldots, n\}\right.$, où $x_{i}$ sont des éléments arbitraires dans $E$ forment un système fondamental de voisinages pour la topologie faible dans $E^{\prime}$. Appelons $k$ l'enveloppe convexe fermé des points $\pm x_{i}(i=1, \ldots, n)$ et soit $h(y)$ la fonction d'appüi de $k$. Etant donné que la fonction d'appui d'un ensemble convexe réduit à un point $a \in E$ est $\langle a, y\rangle$ on a selon le théorème 4 $h(y)=\max \left|\left\langle x_{i}, y\right\rangle\right|$. Donc $U=\{y ; h(y)<1\}$.

Soit $H^{i}(y)$ une fonction d'appui qui est continue pour la topologie faible. $H(y)$ étant continu à l'origine, il existe un voisinage $U=\{y ; h(y)<1\}$ tel que $H(y)<1$ pour $y \in U$. Donc on a partout $H(y) \leq h(y)$, c'est-à-dire $K \subset k$. Par conséquent $K$ est borné et de dimension finie.

Inversement soit $K$ borné et de dimension fïnie. Pour un choix convenable des points $x_{i}$ on a $K \subset k$, d'où $H(y) \leq h(y)$. Il s'ensuit de l'inégalité évidente $\left|H\left(y_{1}\right) \div H\left(y_{2}\right)\right| \leq h\left(y_{1}-y_{2}\right)$ que $\left|H\left(y_{1}\right)-H\left(y_{2}\right)\right|<\varepsilon$ si $y_{1}-y_{2} \in \varepsilon U$ et la continuité uniforme de $H(y)$ est démontrée.

Nous pouvons également démontrer un théorème analogue pour la topologie forte:

Théorème 7. La fonction d'appui de $K$ est continue pour la topologie forte si et seulement si $K$ est fortement borné.

La démonstration est aussi analogue à celle du théorème 6 . On remarque d'abord que les fonctions d'appui des ensembles convexes fermés et bornés dans 
$E$, qui sont symétriques autour de l'origine, forment un système de seminormes qui définit la topologie forte dans $E^{\prime}$.

Par la suite nous allons exclusivement étudier la classe $\mathcal{K}$ des ensembles convexes fermés fortement bornés. Ces ensembles sont à fortiori faiblement bornés, donc leurs fonctions d'appui sont finies partout. $\mathcal{K}$ sera muni de la topologie d'Hausdorff, c'est-à-dire pour tout voisinage $U$ de l'origine dans $E$ et $\varepsilon>0$ on' définit un voisinage de $K_{0} \in \mathcal{K}$ par les conditions

$$
K \subset K_{0}+\varepsilon U, K_{0} \subset K+\varepsilon U .
$$

On obtient évidemment un système fondamental de voisinages si $U$ parcourt tous les voisinages convexes symétriques.

Les voisinages peuvent s'exprimer facilement au moyen des fonctions d'appui. En effet, d'après le théorème 2 les conditions (5) sont équivalentes aux inégalités $H(y) \leq H_{0}(y)+\varepsilon V(y), H_{0}(y) \leq H(y)+\varepsilon V(y)$ où $V(y)$ est la fonction d'appui de $U$. Donc les voisinages sont définis par

$$
\sup _{y} \frac{\left|H(y)-H_{0}(y)\right|}{V(y)}=\sup _{V(y) \leq 1}\left|H(y)-H_{0}(y)\right| \leq \varepsilon .
$$

Remarquons que $\{y ; V(y) \leq 1\}$ est la polaire du voisinage $U$ et par conséquent un ensemble équicontinu dans $E^{\prime}$ (voir Dieudonné [3]).

Considérons l'espace vectoriel $\mathcal{H}$ de toutes les fonctions positivement homo. gènes $h(y)$ définies dans $E^{\prime}$, qui sont continues pour la topologie forte, et introduisons dans $\mathcal{H}$ la topologie de convergence uniforme sur tout ensemble équicontinu. Etant donné qu'un ensemble équicontinu est fortement borné, on voit de suite que $\mathcal{H}$ est un espace vectoriel topologique localement convexe.

Les fonctions d'appui des ensembles convexes $K \in \mathcal{K}$ sont dans $\mathcal{H}$ (théorème 7) et la convergence dans $\mathcal{K}$ équivaut à la convergence des fonctions d'appui dans $\mathcal{H}$. Donc les théorèmes 1-3 peuvent s'énoncer comme il suit:

Théorème 8. L'application $\mathcal{K} \ni K \rightarrow H(y) \in \mathcal{H}$ est un isomorphisme des structures algébriques et topologiques et aussi de la relation d'ordre de $\mathcal{K}$ dans $\mathcal{H}$.

Ceci est une généralisation du théorème de Rådström. On remarque que la démonstration n'utilise rien du théorème 5 , le seul théorème difficile.

Soit $\mathcal{H}_{0}$ le sous-espace vectoriel de $\mathcal{H}$ qui est engendré par les fonctions d'appui, autrement dit, les éléments de $H_{0}$ sont de la forme $H_{1}-H_{2}$ où $H_{1}$ et $H_{2}$ sont des fonctions d'appui.

Théorème 9. $\mathcal{H}_{0}$ est réticulé, c'est-à-dire si $h_{1}$ et $h_{2}$ appartiennent à $\mathcal{H}_{0}$ les fonctions $\sup \left(h_{1}, h_{2}\right)$ et inf $\left(h_{1}, h_{2}\right)$ sont dans $\mathcal{H}_{0}$ elles aussi. $\mathcal{H}_{0}$ sépare deux points quelconques dans $E^{\prime}$ qui ne sont pas des multiples positifs l'un de l'autre.

Posons $h_{1}=H_{1}^{+}-H_{1}^{-}, h_{2}=H_{2}^{+}-H_{2}^{-}$où $H_{1}^{+}, \ldots, H_{2}^{-}$sont des fonctions d'appui. On a

$$
\sup \left(h_{1}, h_{2}\right)=\sup \left(H_{1}^{+}-H_{1}^{-}, H_{2}^{+}-H_{2}^{-}\right)=\sup \left(H_{1}^{+}+H_{2}^{-}, H_{2}^{+}+H_{1}^{-}\right)-\left(H_{1}^{-}+H_{2}^{-}\right) .
$$

Les sommes écrites dans cette formule sont des fonctions d'appui (théorème 3) 


\section{HönmaNDER, Sur la fonction d'appui}

de même que le suprémum (théorème 4 ) et par conséquent sup $\left(h_{1}, h_{2}\right) \in \mathcal{H}_{0}$. La seconde partie du théorème est évidente.

Si la dimension de $E$ est finie on en conclut selon le théorème de StoneWeierstrass, que $\mathcal{H}_{0}$ est dense dans $\mathcal{H}$. Dans le cas d'une dimension infinie ceci n'est pas vrai en général. Par exemple si $E$ est un espace normé non réflexif on a $\overline{\boldsymbol{H}}_{0} \neq \boldsymbol{H}$.

Ajoutons enfin quelques mots sur le cas particulier où $E$ est un espace normé. La topologie de Hausdorff est alors une métrique et la distance $d\left(K_{1}, K_{2}\right)$ est par définition la limite inférieure des nombres $t>0$ pour lesquelles on a

$$
K_{1} \subset K_{2}+t S, K_{2} \subset K_{1}+t S,
$$

où $S$ est la boule unité, $S=\{x ;\|x\| \leq 1\}$. La fonction d'appui de $S$ est $\|y\|$, donc la distance peut s'écrire:

$$
d\left(K_{1}, K_{2}\right)=\sup _{y} \frac{\left|H_{1}(y)-H_{2}(y)\right|}{\|y\|}=\sup _{\|y\| \leq 1}\left|H_{1}(y)-H_{2}(y)\right| .
$$

$\mathcal{H}$ est maintenant l'espace normé et complet des fonctions $h(y)$ positivement homogènes qui sont continues pour la topologie forte, et la norme dans $\mathcal{H}$ est définie par la formule

$$
\|h\|=\sup _{\|y\| \leqslant 1}|h(y)|
$$

Donc l'ensemble $\mathcal{K}$ est isomorphe à un cône convexe dans un espace normé.

\section{B I B L I O G R A P H I E}

[1] Bourbaki: Espaces vectoriels topologiques Chap. I, II, Paris 1953.

[2] Dieudonné: La dualité dans les espaces vectoriels topologiques, Ann. Sc. Ec. Norm. Sup. $3^{e}$ sér. 59, 1942, 107-139.

[3] - - Recent developments in the theory of locally convex vector spaces, Bull. of the Amer. Math. Soc. 59 (1953), 495-512.

[4] RAdström: An embedding theorem for spaces of convex sets, Proc. of the Amer. Math. Soc. $3,1952,165-169$.

[5] SANDGRen: On convex cones. À paraître dans Mathematica Scandinavica.

Tryckt den 6 oktober 1954

Uppsala 1954. Almquist \& Wiksells Boktryckeri AB 